\title{
UNDER WATER NOISE REDUCTION USING WAVELET AND SAVITZKY-GOLAY
}

\author{
Selva Balan ${ }^{1}$,Arti Khaparde ${ }^{2}$,Vanita Tank ${ }^{2}$,Tejashri Rade ${ }^{2}$ and Kirti \\ Takalkar $^{2}$ \\ ${ }^{1}$ Central Water and Power Research Station, \\ Khadakwasla, Pune, Maharashtra, India \\ ${ }^{2}$ Department of E \&TC, Maharashtra Institute of Technology, Pune, \\ Maharashtra, India
}

\begin{abstract}
A precise, linear indication of the depth of water in a specific part of water body is what always required. Presently there are a wide variety of ways to produce a signal that tracks the depth of water.The Ultrasonic signal is most commonly used for the depth estimation. This signal is affected by various underwater noises which results in inaccurate depth estimation. The objective of this paper is to provide noise reduction methods for underwater acoustic signal.In present work, the signal processing is done on the data collected using TC2122 dual frequency transducer along with the Navisound 415 echo sounder. There are two signal processing techniques which are used: The first method is denoising algorithm based on Stationary wavelet transform (SWT)and second method is Savitzky-Golay filter. The results are evaluated based on the criteria of peak signal to noise ratio and $3 D$ Surfer plots of the dam reservoir whose depth estimation has to be done.
\end{abstract}

\section{KEYWORDS}

Savitzky-Golay filter, Wavelet transform, PSNR

\section{INTRODUCTION}

Dams in any country are a part of several multi-purpose projects to serve a variety of needs. Basically, dams are built to harness the river water so that it can be utilized according to the needs. A multipurpose project is often launched for storing water for irrigation purposes, providing drinking water and generating hydro-electricity. The water stored by the dams can also be used to prevent floods and facilitate forestation in the catchments areas of the reservoirs. Depth measurement of water bodies has developed remarkably in the last few decades with the adaptation of new techniques and technologies. Several methods have been studied and introduced by researchers for assessing the depth of the reservoirs using Ultrasonic signal.

Ultrasound wave is basically cyclic sound pressure whose frequency ranges from $15 \mathrm{kHz}$ to 200 $\mathrm{kHz}$ [1]. The depth measurement is quite sensitive to variations of the sound velocity profile. The sound velocity profile is affected by factors such as, variation of one degree Celsius in temperature, salinity which is a measure of the quantity of dissolved salts and other minerals in water and the total amount of dissolved solids in water. The pressure also has a significant impact on the sound velocity variation and has a major influence on the sound velocity in deep water [2].

Dhinaharan Nagamalai et al. (Eds) : CSE, DBDM, CCNET, AIFL, SCOM, CICS, CSIP- 2014 
When an ultrasonic wave is transmitted through water, it is expected to reach the bottom and then reflect back, but instead of this, it gets contaminated with noise and gets reflected back by the obstacles such as stones, waste thrown in water or the creatures living under the water. This gives a false bottom anticipation which doesn't provide the accurate results.

This paper deals with depth analysis of the water reservoir using reflected ultrasound waves. The bed of the dam is mostly even due to deposition of silt by the water current. When the reflected signal of sensor is plotted in a 3D image, it is shown as a bed containing sharp peaks which is not expected at the bottom. These sharp peaks could be the reflections from the suspended obstacles which come in the path of the transmitted ultrasonic signal.

The data was collected using sensor Reson's TC2122 dual frequency survey echo sounder transducer which works on two resonant frequencies $33 \mathrm{kHz}$ and $200 \mathrm{kHz}$ and Reson's Navisound 415 hydrographic single beam echo sounder.General assumption is that the noise present is white Gaussian noise but the underwater noise does not full fill the classical white noise assumption [3] and hence Non-white noise is assumed. To reduce noise from the given data and to estimate approximate depth, two techniques are applied- Denoising based on Stationary Wavelet Transform and Savitzky-Golay filter.

This paper is organized as follows:-Section 2 deals with wavelet transforms. Savitzky-Golay filter is explained in section 3 , Section $4 \&$ Section 5 deal with results \& conclusion respectively.

\section{WAVELET TRANSFORM}

Wavelet transforms have become one of the most important and powerful tool for signal denoising [4]. Discrete Stationary Wavelet Transform is undecimated versions of discrete wavelet transform which is used for signal denoising [5] and pattern recognition. The main idea is to average several detailed coefficients which are obtained by decomposition of the input signal [6].

There are a number of wavelets that can be used for noise removal: Harr, Daubechies, Symlet, Coiflet, Biorthogonal, Reverse Biorthogonal, Meyer [7][8] to name few. In order to use the wavelet transform effectivelythe details of the particular application should be taken into account and the appropriate wavelet should be chosen. They are chosen based on their shape and their ability to analyze signal in particular application [9]. The performance of wavelet based denoising depends on wavelet decomposition structure.

For selecting particular type of wavelet, performance comparison of some known wavelet familieswas done and their effect on the given signalwas observed.In present case, as explained earlier smoothness of the surface is the basic criteria for depth estimation, so accordingly one wavelet from each wavelet family was selected. These are shown in Table 1.

Table 1.Wavelet selected from respective wavelet family.

\begin{tabular}{|l|l|}
\hline Wavelet Family & Selected wavelet \\
\hline Harr & harr \\
\hline Daubechies & db8 \\
\hline Symlet & sym5 \\
\hline Coiflet & coif5 \\
\hline Meyer & dmey \\
\hline Biorthogonal & bior2.2 \\
\hline Reverse biorthogonal & rbior2.2 \\
\hline
\end{tabular}


The detailed and approximation coefficients are obtained using signal decomposition. Further decomposition of approximation coefficients up to specified level is done.The maximum decomposition level depends on number of data points contained in a data set. Present depth analysis 5 decomposition levels were found to be appropriate.

Thresholding of data in wavelet domain is done to smooth out or to remove some of the coefficients of wavelet transform of measured sub-signal introduced due to noise or obstacles in water bodies [10]. Two commonly used types of thresholding are hard and soft thresholding. In hard thresholding [10][11] if any coefficient $(\mathrm{x})$ less than threshold value $(\mathrm{t})$ then it is set to zero otherwise it remains unchanged.

$$
\operatorname{hard}(x)=\left\{\begin{array}{l}
x ; \quad|x|>t \\
0 ; \text { otherwise }
\end{array}\right.
$$

Soft thresholding [10][11] is similar to hard thresholding with a little difference i.e. no coefficient remains unchanged instead it is shrunken by threshold value(t). The present analysis is done using soft thresholding technique.

$$
\operatorname{soft}(x)=\left\{\begin{array}{l}
x-t ;|x|>t \\
0 ; \text { otherwise }
\end{array}\right.
$$

\section{SAVITZKY-GOLAY FILTER}

The Savitzky-Golay filter is a particular type of low-pass filter. It is well-adapted for data smoothing. It is also referred to as least-squares or Polynomial Smoothing filter [12]. Rather than having their properties defined in the Fourier domain, and then translated to the time domain, Savitzky-Golay filters derive directly from a particular formulation of the data smoothing problem [7] in the time domain. These filters are of type-I FIR low pass filters with nominal pass band gain of unity [14].Savitzky and Golay proposed the method of data smoothing based on local least-squares polynomial approximation [14]. Polynomial smoothing is the process which replaces the noisy samples by the values that lie on the smooth polynomial curves drawn between the noisy samples. For every polynomial order, the coefficients must be determined optimally such that the corresponding polynomial curve best fits the given data [12]. Instead of applying averaging filter it is better to perform least squares fit of a small set of consecutive data points to a polynomial. So Least-squares fit technique is used to choose the polynomial coefficients such that they give minimum mean square error [12][15]. The output smoothed value is taken at the center of the window to replace the original data.

In Savitzky-Golay filter, the odd-indexed coefficients of the impulse response design polynomial are all zero. The nominal normalized cutoff ( $3 \mathrm{~dB}$ down) frequency depends on both the implicit polynomial order and the length of the impulse response. The impulse response of filter is symmetric,so the frequency response is purely real. These filters have very flat frequency response in their pass bands and fair attenuation characteristics in their stop band regions [14].

Following are the constraints on polynomial fitting [14]

- The number of data points must be strictly greater than the number of undetermined coefficients to achieve smoothing by the Savitzky-Golay process.

- If the order of the polynomial is too large, the solution will be of no value.

Generalize algorithm is as follows:

Consider frame size or filter length $N$ is odd, $N=2 M+1$ and $N \geq d+1$ where $d$ is order of polynomial. 
If $x$ is noisy signal with noisy samples $x_{n}, n=0,1, \ldots \ldots . ., L-1$ and it is supposed to be replaced by its smoothed output version $y$ which contains $y_{n}, n=0,1, \ldots . . ., L-1$ then input vector has $n=L$ input points and $x=\left[x_{0}, x_{1} \ldots x_{L-1}\right]^{T}$ is replaced by $N$ dimensional one, having $M$ points on each side of $x$.

$x=\left[x_{-M}, \ldots x_{-1}, x_{0}, x_{1}, \ldots, x_{M}\right]^{T}$

There are 3 cases, for calculating the output result. These cases are explained in [16]. Smoothed output $\mathrm{y}$ is calculated as

$y=B x$

The Savitzky-Golay filter coefficients $b_{0}, b_{1}, \ldots$...are the elements of matrix $B$.

$B=\left[b_{-M}, \ldots . b_{-1}, b_{0}, b_{1}, \ldots . b_{M}\right]$

$B=G S^{T}$

Where $G=S\left(S^{T} S\right)^{-1}, \quad S=\left[s_{0}, s_{1}, \ldots, s_{d}\right], \quad s_{0}(m)=1, s_{1}(m)=m, s_{2}(m)=m^{2}, \ldots, s_{d}(m)=$ $m^{d}$ where $-d \leq m \leq d[12][13]$.

\section{RESULTS AND DISCUSSION}

The peak signal to noise ratio represents the measure of peak error [9]. It is given as,

$$
P S N R=10 \log _{10}\left(\frac{R^{2}}{M S E}\right) d B
$$

Where

$M S E=\frac{1}{m} \sum_{i=1}^{m}[I(i)-O(i)]^{2}$

$M S E$ is Mean Square Error

$I=$ original value

$O=$ output value

$R=$ maximum input value

Generally PSNR should be greater than 30dB in order to reduce noise effectively.

For comparing results of Savitzky-Golay filter, another parameter used is Time Constraints which is time required for execution of program.

Table 2. Values of PSNR for different types of wavelets.

\begin{tabular}{|c|c|c|c|c|c|c|c|}
\hline FileNo. & Harr & Bior & Rbior & Sym & Coif & Dmey & Daub \\
\hline 1 & 41.93 & 41.79 & 42.04 & 41.22 & 40.66 & 40.48 & 40.46 \\
\hline 2 & 51.74 & 50.51 & 49.41 & 49.25 & 48.68 & 48.34 & 48.26 \\
\hline 3 & 43.79 & 43.77 & 43.23 & 43.02 & 42.73 & 42.59 & 42.78 \\
\hline 4 & 43.98 & 43.59 & 42.84 & 43.02 & 42.96 & 42.86 & 42.59 \\
\hline 5 & 40.07 & 39.24 & 39.17 & 39.89 & 39.03 & 38.59 & 39.76 \\
\hline 6 & 37.07 & 37.34 & 37.07 & 36.87 & 36.84 & 36.77 & 36.90 \\
\hline 7 & 40.00 & 40.37 & 40.39 & 39.77 & 39.80 & 39.64 & 39.91 \\
\hline
\end{tabular}


The results presented in Table 2 show PSNR values for different wavelets. It can be seen that Harr wavelet is giving better result than other wavelets in this case.

Table 3. Values of PSNR by varying order and with fixed frame size for Savitzky-Golay filter.

\begin{tabular}{|c|c|c|c|c|}
\hline File & Or1_31 & Or2_31 & Or3_31 & Or4_31 \\
\hline File1 & 40.6843 & 42.5433 & 42.6120 & $\mathbf{4 4 . 0 2 8 0}$ \\
\hline File2 & 46.7581 & 47.8004 & 47.7967 & $\mathbf{4 9 . 4 8 0 8}$ \\
\hline File3 & 42.0438 & 43.0886 & 43.1009 & $\mathbf{4 4 . 4 3 6 7}$ \\
\hline File4 & 42.0823 & 43.8442 & 43.8360 & $\mathbf{4 4 . 7 8 2 4}$ \\
\hline File5 & 39.0119 & 39.7840 & 39.7839 & $\mathbf{4 0 . 8 5 6 6}$ \\
\hline File6 & 36.4540 & 36.7830 & 36.8013 & $\mathbf{3 7 . 2 9 3 0}$ \\
\hline File7 & 39.2207 & 40.6914 & 40.7429 & $\mathbf{4 1 . 1 4 0 4}$ \\
\hline Avg. Time(sec) & 2.59 & 2.41 & 2.62 & $\mathbf{2 . 4 1 9 8 1}$ \\
\hline
\end{tabular}

From Table 3 it can be seen that as the order of polynomial increases, PSNR value also increases. So PSNR is directly proportional to order of polynomial for Savitzky-Golay filter. Computational complexity is less for higher order. (Processer used-Intel core i5)

Table 4. Values of PSNR by varying frame size and with fixed order for Savitzky-Golay filter

\begin{tabular}{|c|c|c|c|c|}
\hline File & Or4_31 & Or4_33 & Or4_41 & Or4_49 \\
\hline File1 & $\mathbf{4 4 . 0 2 8 0}$ & 43.8143 & 43.0032 & 42.6637 \\
\hline File2 & $\mathbf{4 9 . 4 8 0 8}$ & 49.1640 & 48.2272 & 47.8468 \\
\hline File3 & $\mathbf{4 4 . 4 3 6 7}$ & 44.0604 & 43.4561 & 43.1529 \\
\hline File4 & $\mathbf{4 4 . 7 8 2 4}$ & 44.6722 & 44.1377 & 43.7461 \\
\hline File5 & $\mathbf{4 0 . 8 5 6 6}$ & 40.6501 & 40.0252 & 39.9840 \\
\hline File6 & $\mathbf{3 7 . 2 9 3 0}$ & 37.0813 & 37.0102 & 36.8819 \\
\hline File7 & $\mathbf{4 1 . 1 4 0 4}$ & 41.1594 & 40.9853 & 40.5424 \\
\hline Avg.Time (sec) & $\mathbf{2 . 4 1}$ & 2.55 & 2.53 & 2.56 \\
\hline
\end{tabular}

From Table 5 it can be seen that as the lesser the frame size, more is the PSNR. So PSNR is inversely proportional to frame size for Savitzky-Golay filter. Computational complexity is less for smaller frame size.(Processer used-Intel core i5)

3D plots of signal are obtained using software surfer11 which are shown below:

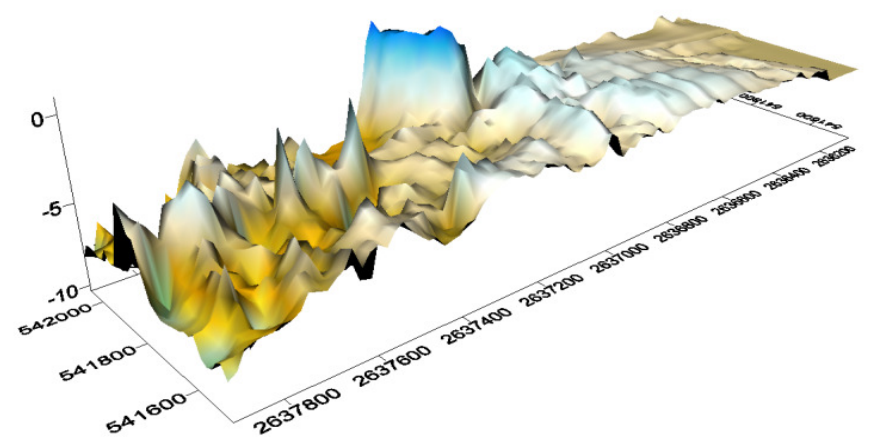

Figure 1.Original signal 


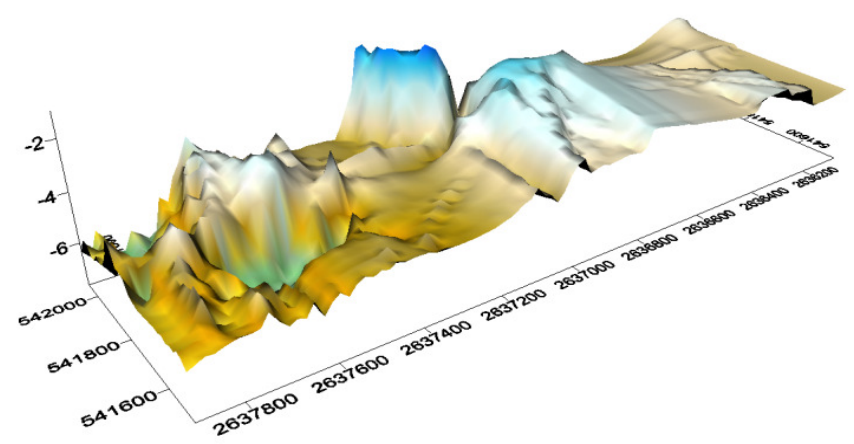

Figure 2. Signal processed using Harr wavelet

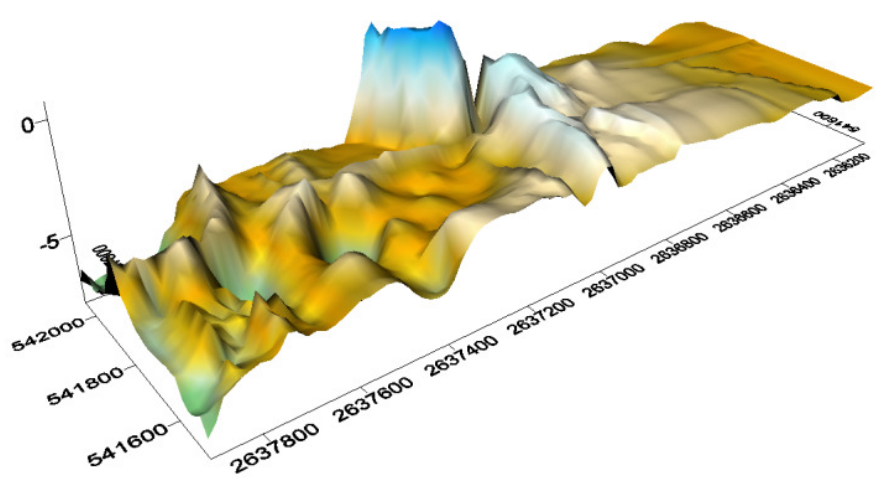

Figure 3.Signal processed using Savitzky-Golay Filter

\section{CONCLUSION}

Henceanalysis of Ultrasonic signal is done using two techniques:Harr wavelet Transform and Savitzky-Golay filter.It is found that out of all wavelet transforms, harrwavelet is most suitable for noise reduction in ultrasonic signal because PSNR value is high among all the wavelets used. The present paper gives a good comparison of wavelet based denoising of acoustic data.

In Savitzky-Golay Filter, analysis is done for different orders of polynomial and frame sizes which show that with higher order of polynomial and lesser frame size PSNR is high.

The results from surfer plots show that the harr wavelet with decomposition level up to 5 and Savitzky-Golay filter with order 4 and frame size 31 can be effectively used for smoothing the data obtained which can lead to estimation of depth with minimum error using empirical formula designed for a particular application.

\section{ACKNOWLEDGEMENT}

We are thankful to Central Water and Power Research Station for providing the data of reservoir in India. 


\section{REFERENCES}

[1] Sabuj Das Gupta, Islam Md. Shahinur, Akond Anisul Haque, Amin Ruhul, Sudip Majumder,(October 2012)"Design and Implementation of Water Depth Measurement and Object Detection Model Using Ultrasonic Signal System",International Journal of Engineering Research and Development, Volume 4, Issue 3, pp.62-69.

[2] International hydrographic Bureau, (2005)"Manual on hydrography", M-13, pp.126.

[3] Arnaud Jarrot, Cornel Ioana, Andr'e Quinquis, (2005)"Denoising Underwater Signals Propagating Through Multi-path Channels", Oceans - Europe (Volume:1) pp.501-506.

[4] SJS Tsai, (2002)"Chapter 4 Wavelet Transform and Denoising".

[5] Chu-Kueitu, Yan-Yao Jang, (2004)"Development of Noise Reduction Algorithm for Underwater Signals", Underwater Technology, International Symposium on, pp.175-179.

[6] V. Matz and J. Kerka, "DIGITAL SIGNAL PROCESSING OF ULTRASONIC SIGNALS", pp.3.

[7] wavelets.pybytes.com by Filip Wasilewski.

[8] M. Kreidl, P. Houfek, (2002)"Reducing Ultrasounic Signal Noise by Algorithms based on Wavelet Thresholding", Acts Polytechnica Vol. 42, pp.60-65.

[9] S.Kumari, R.Vijay, (January 2012)"Effect of Symlet Filter Order on Denoising of Still Images", Advanced Computing :An International Journal(ACIJ).Vol.3.No.1, pp.137-143.

[10] K.Mathan Raj, S.Sakthivel Murugan, V. Natarajan, S.Radha, (2011)"Denoising Algorithm using Wavelet for Underwater Signal Affected by Wind Driven Ambient Noise", Recent Trends in Information Technology (ICRTIT), pp.943-946.

[11] Bernhard Wieland, (October 2009) "Speech Signal Noise Reduction with Wavelets", pp.55-56.

[12] Sophocles J. Orfanidis, (2010)"Introduction To Signal Processing", Pearson Education, Inc., pp.427451.

[13] William H. Press, Brian P. Flannery, Saul A. Teukolsky, William T. Vetterling, (19881992)"Numerical Recipes in C:The Art of Scientific Computing", Cambridge University Press, pp.650-651.

[14] Ronald W. Schafer, (July 2011)"What is a Savitzky-Golay filter?", IEEE SIGNAL PROCESSING MAGAZINE, pp.111-115.

[15] Savitzky A., and Golay, M.J.E. (1964)"Analytical Chemistry", Volume 36, pp.1627-1639.

[16] Md. Abdul Awal, Sheikh Shanawaz Mostafa and Mohiuddin Ahmad, (2011)"Performance Analysis of Savitzky-GolaySmoothing Filter Using ECG Signal", IJCIT, VOLUME 01 ISSUE 02, pp.24-29.

\section{Authors}

Mr. Selva Balan is currently working as a Chief Research Officer at Central Water and Research Power Station (CWPRS), Khadakwasla, Pune, India. At CWPRS he is associated with various national important projects under hydrology projects, development projects related to Ports, Coastal development, River Engineering, Dam Instrumentation, Remote sensing, Real time systems and many other projects. He is Faculty at National Water Academy.Published more than 50 conference, 20 journal papers. He is an Expert Member nominated under WORLD BANK projects in hydrology group.He is an Editor for International Standards on Hydrometry, and at BIS. His research interests include ANN, Fuzzy logic, Microcontrollers, Image processing, Wavelets etc

Dr. Arti Khaparde is currently working as Professor in the Department of Electronics and Telecommunication with Maharashtra Institute of Technology, Pune. She has vast experience of 14 years in teaching and research and almost 20 Publications on her name in reputed International Conferences and Journals. She received her Ph.D. in Electronics and Communication Engineering, from JNTU, Hyderabad in 2009. Her research interests include Digital Signal processing, noise removal techniques, Digital Image and video processing, Pattern recognition, Programming of microprocessors and microcontrollers.

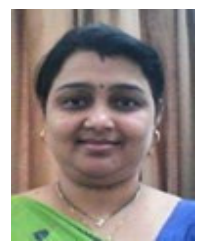


Ms. Vanita Tank received her B.E degree from Barktullah University and M.Tech from Maulana Azad National Institute of Technology, Bhopal. Currently she is working as Assistant Professor in the Department of Electronics and Telecommunication with Maharashtra Institute of Technology, Pune and pursuing her Ph.D in audio and speech signal processing from University of Pune. She has 7 years of experience in teaching. Her current interest includes applications of signal processing and signal coding.

Ms. Tejashri Rade is a final year student of B.E Electronics \& Telecommunication in Maharashtra Institute of Technology, Pune. Presently she is working under Mr. Selva Balan and Ms. Vanita Raj Tank for her final year project on Noise reduction from Ultra sound waves.

Ms. Kirti Takalkar is a final year student of B.E Electronics \& Telecommunication in Maharashtra Institute of Technology, Pune. Presently she is working under Mr. Selva Balan and Ms. Vanita Raj Tank for her final year project on Noise reduction from Ultra sound waves.
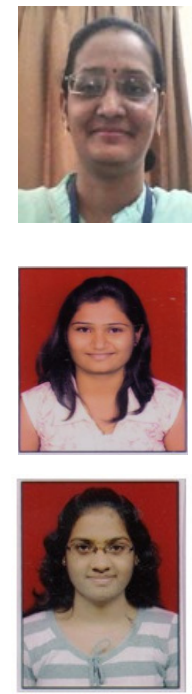